\title{
Vitamin D Deficiency Presenting as Proximal Myopathy: An Overlooked Diagnosis - A Case Series and Review of the Literature
}

\author{
Authors: $\quad$ *Venkatraman Rajkumar \\ Banting Clinic, India \\ *Correspondence to6abdoctor@gmail.com \\ Disclosure: $\quad$ The author has disclosed no conflicts of interest. \\ Received: $\quad 17.11 .21$ \\ Accepted: $\quad 14.01 .22$ \\ Keywords: $\quad$ Adult coeliac disease, intact parathyroid hormone (iPTH), intramuscular vitamin D, \\ proximal myopathy, 25 hydroxyvitamin D. \\ Citation: $\quad$ EMJ. 2022; DOI/10.33590/emj/21-00248.
}

\section{Abstract}

The prevalence of vitamin D deficiency is ubiquitous. Severe disease can present very dramatically and can be misleading to the treating physician, resulting in mismanagement. A high index of suspicion in vulnerable patients should help circumvent this problem, including a thorough history and physical examination to lead the clinician in the right direction. Insidious onset and progressive proximal pelvic girdle myopathy, in the absence of other neurological findings, in the appropriate patient should prompt the physician to test for serum 25 hydroxyvitamin D levels.

The literature on endocrine myopathy does not highlight this condition as much as it does the thyroid, parathyroid, and adrenal aetiologies. Testing for vitamin D deficiency is simple, and treatment is available all over the world. The following article presents three patients, in different scenarios, with the condition. In all three patients, incapacitating pelvic girdle weakness resolved and quality of life improved dramatically with timely intervention. Most importantly, the correct approach prevented misdiagnosis and mismanagement.

\section{INTRODUCTION}

Vitamin $D$ is essential for skeletal and skeletal muscle health. It is present in varying quantities in certain foods, but the average diet does not contain enough to meet daily needs. A good source is exposure to sunlight for sufficient time to enable the skin to synthesise the vitamin from cholesterol using the ultraviolet spectrum. In some countries, a regulated process of fortification

of certain commonly consumed foods exists. Vitamin $D$ deficiency is found all over the world, even in sunnier, more tropical countries. Factors contributing to this deficiency includes poor food choices, malnutrition, malabsorption, extreme obesity, religious prohibitions mandating people to cover the bodily areas, work schedules predominantly indoors, the use of P450 enzyme-inducing medications, chronic kidney disease, and lack of awareness of the condition, even among healthcare professionals. 
To identify at-risk individuals, diet analysis, sun exposure time, and investigating malabsorption states are crucial. A growing number of patients developing multiple vitamin and mineral deficiencies after bariatric surgery bypasses are a recent addition. ${ }^{2}$ Timely intervention can reduce morbidity and mortality to a great extent. Furthermore, prevention of this condition is most cost-effective.

Early on, clinically asymptomatic deficiency is common. As the disease progresses, bone pain, muscle aches, and muscle weakness sets in. During this phase, as a result of the non-specific nature of the symptoms, the diagnosis is often overlooked. As the condition progresses, patients may develop profound weakness in the proximal muscles of the pelvic girdle, which leads to difficulty in rising from a bed, chair, or climbing stairs. Patients may become bedridden and need multi-person assistance for their activities of daily living ( $A D L$ ), compromising their quality of life. A high index of suspicion in patients presenting with pelvic girdle myopathy, in the absence of other neuromuscular findings, will lead to the correct diagnosis. This article illustrates three different case scenarios, all presenting with proximal myopathy and clinical confusion with other myopathic disorders or malignancies that can lead to disastrous consequences.

\section{CASE PRESENTATIONS}

\section{Patient One}

A 60-year-old female and entrepreneur of Indian descent presented with an inability to raise themselves from bed for 1 month. They had been bedridden and required the assistance of two people to perform their ADL. Their problem started 8 months earlier, with difficulty climbing stairs and became progressively worse, forcing them to shift their office to the ground level. There was no improvement after 2 months and they were finding it difficult to rise from their chair. The weakness progressed and they became bedridden. In addition, they experienced generalised pains in their muscles and bones that, at times, disturbed their sleep.

Their past medical history was significant for well-controlled hypertension, while their surgical and social history were not contributory. They had natural menopause 8 years ago and is not on hormone replacement. A review of systems was not contributory. One morning, they woke up with severe pain in their right hip and presented to an orthopaedic surgeon, fearing a fracture. The orthopaedist evaluated them and ordered X-rays, finding extreme osteopenia but no fractures. The patient had a raised serum alkaline phosphatase and low normal calcium and phosphate. A neurologist entertained a diagnosis of myopathy. The neurologist asked for an endocrinologist to see her.

The consultant noted that the patient had not received sunshine for almost a year. On examination, the consultant found the patient to be well-nourished without pallor. Their blood pressure was well controlled. Neurological examination was significant for the severe weakness of the muscles of the hip and lower back but otherwise did not reveal any abnormalities.

The endocrinologist ordered a serum of 25 hydroxyvitamin D (25[OH]D) and intact PTH (iPTH) levels. The former was less than 1.0 $\mathrm{ng} / \mathrm{mL}$ and the latter was $488.0 \mathrm{pg} / \mathrm{mL}$. A diagnosis of secondary hyperparathyroidism due to vitamin D deficiency was evident.

The patient received an intramuscular injection of vitamin D3 600,000 IU and calcium tablets of $1,000 \mathrm{mg}$ elemental calcium per day. A nutritionist counselled them on increasing their dietary calcium. The patient started noticing an improvement in their strength. Gradually, they progressed to getting up from bed on their own. In 4 weeks, they walked without support into the endocrinologist's office. Subsequently, the $25(\mathrm{OH}) \mathrm{D}$ level was $38 \mathrm{ng} / \mathrm{mL}$ and iPTH was $166 \mathrm{pg} / \mathrm{mL}$. they continued to receive oral vitamin D 1,000 IU daily and adequate dietary calcium.

\section{Patient Two}

A 70-year-old male, a retired postal employee, presented with an inability to stand from their chair for 4 months. They were very active during employment and after retirement 10 years previous. They had no significant past medical, surgical, or social history. One year ago, the patient's partner became bedridden. Their partner had been diagnosed with breast cancer and widespread bony metastasis. The paitent 
spent most of their time indoors, caring for their partner. The patient paid little attention to themselves and became very depressed when their partner died 6 months before presentation. The patient's weakness prevented their selfcare. They also started getting severe bone pains and muscle aches. Fearing that they had an underlying malignancy, they approached their physician. The internist diagnosed grief but, under pressure from the patient, investigated their bone pains with a nuclear bone scan. The results were shocking: multiple symmetrical hot areas throughout the entire skeleton-rule out metastasis.

An oncologist evaluated the patient, found an enlarged prostate gland consistent with the patient's age but without nodules or induration. The serum prostate specific antigen levels were within limits and no other primary cancer was evident after extensive investigations. An endocrine consultation led to the diagnosis of vitamin D deficiency (serum levels of $25(\mathrm{OH}) \mathrm{D}$ and $\mathrm{iPTH}$ were $2.8 \mathrm{ng} / \mathrm{mL}$ and $366.0 \mathrm{ng} / \mathrm{mL}$, respectively). The patient received an intramuscular injection of vitamin D 600,000 IU once as well as daily calcium supplements. They also received nutritional education for increasing calcium in their diet.

In 4 weeks, their weakness dramatically improved. They no longer found it difficult to get up from their chair. They ambulated without assistive devices and was able to perform all their ADLs. Four months later, a repeat nuclear bone scan showed that most of the hotspots had disappeared. It reassured the treating physicians that they had progressed in the right direction.

The repeat serum $25(\mathrm{OH})$ D and iPTH levels were $39 \mathrm{ng} / \mathrm{mL}$ and $144 \mathrm{pg} / \mathrm{mL}$, respectively. They received 1,000 IU oral vitamin $D$ daily as a maintenance dose and was encouraged to consume calcium-rich foods. After all, the patient did not have malignancy.

\section{Patient Three}

A 36-year-old female software professional, with a history of autoimmune hypothyroidism on levothyroxine $100 \mathrm{mcg}$ daily presented with multiple problems. A year previously, the patient received an out-of-turn promotion for their excellent work performance and assigned to a country where food items were fortified with all vitamins and minerals.

At that time, they noticed muscle aches, fatigue, migraine-like headaches, recurrent painful mouth ulcers, upper respiratory allergies, wheezing, and disturbed sleep. Soon, their menstrual periods started becoming irregular but there was no menorrhagia or clots. Their headaches became more frequent, and their respiratory complaints worsened. They became intolerant to dairy products, with abdominal bloating, cramps, and increased stool frequency. Unsteadiness upon standing with eyes open and closed followed. They started having increasing weakness in their hips and rising from their chair was an ordeal.

The patient's physician had treated them symptomatically for their ailments, without much relief. They met with several specialists, underwent many investigations, and received multiple diagnoses without avail. They presented to a new physician for a fresh look at their problems, who found them adequately nourished without pallor or clinically euthyroid, but significantly anxious and depressed. Neurological examination was positive for the weakness of the muscles of the hip and lower back and mild ataxia.

The new physician reviewed the patient's latest test results and found a normal serum thyroidstimulating hormone level, an elevated red cell distribution width in their haemogram, and a high alkaline phosphatase but normal transaminases. The physician ordered iron studies and $25(\mathrm{OH})$ D. The results were consistent with non-anaemic iron deficiency (low iron, high iron-binding capacity, and decreased ferritin but with normal haemoglobin) and secondary hyperparathyroidism due to vitamin D deficiency (25[OH]D: $8.8 \mathrm{ng} / \mathrm{mL}$; iPTH: $266 \mathrm{pg} / \mathrm{mL}$ ).

The deficiencies of iron and vitamin $D$, with an adequate diet with fortified foods, pointed to gastrointestinal malabsorption. This, in addition to the multiorgan symptomatology, was suggestive of adult coeliac disease. The patient tested positive for serum IgA anti-transglutaminase antibodies, with normal total IgA levels. Since the patient declined an endoscopic duodenal or jejunal mucosal biopsy, histological confirmation was not possible. However, they agreed to try a gluten-free diet. they received oral iron and 
vitamin D (600,000 IU orally once weekly for 8 weeks as initial treatment) and oral calcium supplements. The inability to tolerate dairy products was overcome by adding lactic acid bacillus tablets with each exchange of dairy.

In 4 weeks, the patient was able to get up from the chair without difficulty and most of their other complaints abated and they resumed their work without interruption On follow-up, they required 4,000 IU of vitamin $D$ daily to maintain serum $25(\mathrm{OH}) \mathrm{D}(34 \mathrm{ng} / \mathrm{mL})$ and iPTH levels (41 pg/mL) in the normal range.

\section{DISCUSSION}

\section{Methods of Obtaining Vitamin D}

The Vitamin $D$ is available to the body from two sources. They are the skin and the gastrointestinal tract.

\section{The skin}

The skin has the machinery to synthesise vitamin $D$ from cholesterol using the ultraviolet (UV) spectrum (UV: $290-315 \mathrm{~nm}$

Table 1: Impediments to vitamin D synthesis in the skin.

\begin{tabular}{|l|l|}
\hline Condition & Comments \\
\hline Ageing & Decreased efficiency of synthetic machinery \\
\hline High latitudes & Less UV radiation \\
\hline Times other than midday & Suboptimal UV rays \\
\hline Sunscreens & Even lowest potency products filter UV rays \\
\hline Photosensitivity & Sunlight avoidance \\
\hline Fear/risk of skin cancer & Sunlight avoidance \\
\hline Religious prohibitions & Decreased body surface areas exposed to the sun \\
\hline Cloud cover & Clouds filter UV rays \\
\hline Glass windows & Glass filters UV rays; however, they do let in light and heat \\
\hline Homebound or indoor workplace & Avoidance of sun exposure \\
\hline
\end{tabular}

UV: ultraviolet.

Table 2: Vulnerable groups for vitamin D deficiency.

\begin{tabular}{|l|l|}
\hline Condition & Comments \\
\hline Ageing & Decreased active vitamin D synthesis and intestinal resistance \\
\hline Bedridden & Accompanying poor nutritional states \\
\hline Institutionalisation & Multiple factors \\
\hline Pregnancy & Increased demand \\
\hline Lactation & Increased demand \\
\hline Infants and growing children & Increased demand \\
\hline Obesity & Increased segregation of vitamin D in adipocytes and decreased availability \\
\hline CKD & Decreased active vitamin D synthesis \\
\hline Therapy with p450 enzyme inducers & Increased catabolism of vitamin D \\
\hline Malabsorption states & $\begin{array}{l}\text { Coeliac disease; small intestinal bacterial overgrowth; short bowel due to } \\
\text { disease or surgery; inflammatory bowel diseases; bariatric bypass procedures }\end{array}$ \\
\hline
\end{tabular}


wavelength) of sunlight. The ideal time for sun exposure is midday, when the maximum absorption of UV rays occurs. It is true not only in temperate zones but also in the tropics. The minimal erythemal dose to produce sufficient vitamin D synthesis will depend on the total area and time of skin exposed. ${ }^{3}$ Excess vitamin $D$ synthesised in the skin is metabolised to inactive compounds. Hence, toxicity never occurs by overexposure to sunlight.

Table 1 lists impediments to vitamin D synthesis in the skin.

\section{The gastrointestinal tract}

Some foods, like eggs and fatty fish, contain vitamin D. The liver contains large amounts. However, the average human diet does not meet the daily requirements of vitamin $D$. Hence, if sunlight exposure is not adequate, supplementation is necessary. It is especially true for vulnerable groups (Table 2). Patients who have had bariatric bypass surgery will require a much higher dose of oral supplemental vitamin D. Absorption occurs in the duodenum and upper jejunum. It is protein-mediated at low concentrations as in the diet, and by unregulated passive diffusion at higher concentrations as in pharmacologic dosing. ${ }^{4}$

\section{Metabolism of Vitamin D}

Both sources of vitamin D are bound to the vitamin $\mathrm{D}$ binding protein and is transported to the liver. The next step is 25 hydroxylation in the liver. The final step is 1 hydroxylation in the proximal tubular cells of the kidney. The resultant 1,25 dihydroxy vitamin $D(1,25[\mathrm{OH}] 2 \mathrm{D})$ is an active hormone that binds to the vitamin D receptors (VDR) in many tissues and exerts its actions. Unfortunately, 24 hydroxylation leads to inactivation of both $25(\mathrm{OH})$ $D$ and $1,25(\mathrm{OH}) 2 \mathrm{D}$. The formation of $1,25(\mathrm{OH}) 2 \mathrm{D}$ is catalysed by the enzyme 1-alphahydroxylase, enhanced by PTH. Older adults have decreased 1-alphahydroxylase activity due to diminished renal mass. In the intestinal epithelium, 1,25(OH)2D binds to VDRs and increases calcium-binding protein synthesis, which aids calcium absorption.

\section{Latest Research Revealed}

Adequate vitamin D maintains the (VDR) density in skeletal muscles and, thereby, the number and function of Type 2 fast-twitching skeletal muscle fibres. In addition, through genomic and non-genomic mechanisms, it reduces both inflammatory cytokine induced skeletal muscle damage and intramuscular adiposity by preferential differentiation of common stem cells into myoblasts instead of adipocytes. Therefore, deficiency of vitamin $D$ results is muscle weakness and decreased reaction speed and strength to sudden postural changes and falls are the result. Recent evidence implicates a role of vitamin $D$ in skeletal muscle repair and regeneration., 5

\section{Double Jeopardy in Older Adults}

Older adults are particularly prone to the ill effects of vitamin D deficiency, due to poor synthesis in

Table 3: Comparison of the clinical characteristics of three patients with severe vitamin D deficiency.

\begin{tabular}{|l|l|l|l|}
\hline Patient characteristics & Patient One & Patient Two & Patient Three \\
\hline Age (years) & 60 & 70 & 36 \\
\hline Gender & Female & Male & Female \\
\hline Past sun exposure & None in 1 year & None in 1 year & None in 1 year \\
\hline Food fortification & No & No & Yes \\
\hline Proximal myopathy & ++++ & +++ & ++ \\
\hline Serum alkaline phosphatase* & 747 & 866 & 353 \\
\hline Serum creatine kinase & Normal & Normal & Normal \\
\hline Serum ALT and AST & Normal & Normal & Normal \\
\hline
\end{tabular}


Table 3 continued.

\begin{tabular}{|c|c|c|c|}
\hline Patient characteristics & Patient One & Patient Two & Patient Three \\
\hline Pre-treatment 25(OH)D (ng/mL) & 1 & 2.8 & 8.8 \\
\hline Pre-treatment iPTH $(\mathrm{pg} / \mathrm{mL})^{+}$ & 488 & 366 & 266 \\
\hline Post-treatment $25(\mathrm{OH}) \mathrm{D}(\mathrm{ng} / \mathrm{mL})$ & 38 & 39 & 34 \\
\hline Post-treatment iPTH $(\mathrm{pg} / \mathrm{mL})^{\dagger}$ & 166 & 144 & 41 \\
\hline $\begin{array}{l}\text { Initial vitamin D treatment } \\
\text { modality }\end{array}$ & $\begin{array}{l}\text { IM injection 600,000 IU } \\
\text { once }\end{array}$ & $\begin{array}{l}\text { IM injection 600,000 IU } \\
\text { once }\end{array}$ & $\begin{array}{l}\text { Oral vitamin D sachet } \\
600,000 \text { IU, once weekly for } \\
8 \text { consecutive weeks }\end{array}$ \\
\hline $\begin{array}{l}\text { Maintenance requirements for } \\
\text { vitamin D }\end{array}$ & 1,000 IU orally daily & 1,000 IU orally daily & 4,000 IU orally daily \\
\hline Initial diagnoses & Myopathy & $\begin{array}{l}\text { Bony metastasis, Primary } \\
\text { unknown }\end{array}$ & $\begin{array}{l}\text { Multiple diagnoses: } \\
\text { migraine headaches; upper } \\
\text { respiratory allergy; bronchial } \\
\text { asthma; aphthous ulcers; } \\
\text { irritable bowel syndrome; } \\
\text { anxiety; depression; } \\
\text { fibromyalgia }\end{array}$ \\
\hline Final diagnosis & $\begin{array}{l}\text { Nutritional vitamin D } \\
\text { deficiency }\end{array}$ & $\begin{array}{l}\text { Nutritional vitamin D } \\
\text { deficiency }\end{array}$ & Adult coeliac disease \\
\hline $\begin{array}{l}\text { Recovery of proximal myopathy } \\
\text { with treatment }\end{array}$ & Complete & Complete & Complete \\
\hline
\end{tabular}

*Normal range for serum alkaline phosphatase: 50-150 U/L.

+Normal range for iPTH: $10-65 \mathrm{pg} / \mathrm{mL}$.

25(OH)D: 25 hydroxyvitamin D; ALT: alanine transaminase; AST: aspartate aminotransferase; IM: intramuscular; iPTH:

intact parathyroid hormone.

the skin, diminished 1-alphahydroxylase activity, and resistance to the action of $1,25(\mathrm{OH}) 2 \mathrm{D}$ in the intestines. A low bone mass and skeletal muscle weakness of ageing are worsened by a concomitant vitamin D deficiency. Studies of treatment in institutionalised older adults with severe vitamin $D$ deficiency have conclusively shown a reduced incidence of falls and fractures.

\section{Clinical Course of Vitamin D Deficiency}

Mild deficiency may be asymptomatic. The deficiency is overlooked initially due to the non-specific nature of the symptoms. As the disease progresses, patients may complain of symptoms like muscle pains, fatigue, and weakness. Bone pains occur due to osteomalacia. Later on, severe muscular weakness is a hallmark condition, with a predilection to the proximal musculature of the hip region. The reason for this is unclear. The ensuing weakness may lead to falls and fractures, or the patient may become immobile due to muscle weakness as mentioned earlier. Severe localised pain in one or more bones may be due to an incomplete fracture identified on X-rays as Looser's zones.

\section{The clinical course in context}

See Table 3 for a comparison of the clinical characteristics of the three patients.

Whenever a patient presents with proximal myopathy, it is imperative to consider many conditions in the differential diagnosis. This is dictated by the clinical presentations. The age at initial presentation did not favour hereditary myopathies. The absence of drugs such as statins, corticosteroids, and retroviral drugs excluded drug-induced myopathy. Clinical features 
suggesting exposure to infective agents or toxins were also absent in all three patients. Also, features of connective tissue diseases like systemic lupus erythematosus, dermatomyositis or polymyositis, and inclusion body myositis were absent in all three patients. An extensive search did not reveal a malignancy in Patient Two. Clinically there were no suggestions of hypo- and hyperthyroidism, Cushing's disease, or acromegaly to pursue these conditions by appropriate investigations. Conditions that caused both proximal and distal myopathies, either simultaneously or sequentially, were also excluded as only the hip muscles were affected. For similar reasons, diseases affecting oculofacial and pharyngeal musculature and conditions that cause combined motor and sensory pathologies were not entertained.

All three patients did not have pre-treatment sun exposure and severe muscle weakness was a feature in all of them. This led to misdiagnosis as myopathy in the first patient who also had features of bone loss on X-rays.

\section{Bone pain does not warrant a bone scan}

Indiscriminate use of a nuclear bone scan to investigate bone pains can lead to the erroneous diagnosis of metastatic malignancy, as noticed in Patient Two. The hotspots observed were areas of bone lysis by the elevated PTH of secondary hyperparathyroidism that disappeared after treatment, which was confirmed by a subsequent scan.

\section{Think adult coeliac disease}

The third patient had a complex presentation, challenging even the most astute clinician. Features of a multi-system involvement, predominantly outside the gastrointestinal system, have been described in adult atypical coeliac disease. This patient had a history of autoimmune hypothyroidism, which has known associations with coeliac disease. In addition, biochemical evidence of iron deficiency in the absence of blood loss, along with vitamin D deficiency despite consuming fortified foods strengthened the probability of adult atypical coeliac disease in this patient. For a detailed description of adult coeliac disease, the reader is directed to the cited reference. ${ }^{7}$ This young patient also had a secondary lactose intolerance that responded to lactic acid bacillus given orally. It supplies the deficient galactosidase enzyme to aid in lactose digestion and allows the patient to tolerate dairy products with the benefit of obtaining natural calcium.

\section{Bariatric surgical patients: a special group}

Vitamin D deficiency is an established complication of obesity and its treatment, namely bariatric surgery. The induced malabsorption may require a high maintenance dose of vitamin $D$ and this may not be cost-effective during long-term therapy. ${ }^{8}$ However, the Endocrine Society guidelines only mention the oral formulation.

\section{Parenteral vitamin D: some food for thought}

Intramuscular (IM) injections of vitamin D are an alternative when very high oral doses are required to maintain the $25(\mathrm{OH}) \mathrm{D}$ within the normal range. There are minimal or no side effects but paucity of adequate data to exclude complications such as hypercalciuria indicate that randomised controlled trials are needed to support or refute IM vitamin D for treatment. A small study from Australia reported the safety of IM vitamin D.9 The first two patients described received injections as initial treatment because high dose oral preparations were locally unavailable at that time. Subsequently, they received smaller oral maintenance doses and responded well. The third patient presented when high-dose oral formulations became available and, although suffering from malabsorption, responded well albeit needing a higher dose.

\section{Dosing regimen}

Another issue is whether to choose a daily, weekly, or once monthly regimen for maintenance. All three regimens are effective and, the choice depends on patient characteristics (compliance issues) and provider preferences. ${ }^{10}$

\section{Vitamin D2 or D3? What is the difference?}

Two formulations of vitamin $D$ are available. Vitamin D2 (ergocalciferol) is from plants and Vitamin D3 (cholecalciferol) is from animal sources. Again, the choice of which compound to use will depend on patient preferences (whether they are vegan or vegetarian) or provider choices. The Endocrine Society considers them equipotential but 
some assays of $25(\mathrm{OH}) \mathrm{D}$ may underestimate levels when treating with vitamin D2 and may complicate management."

\section{Follow-up testing}

Serum 25(OH)D normalised in all three patients but iPTH normalised only in Patient Three. The normalisation of iPTH may happen later (sometimes after one or two years). The reasons behind this are not clear.

The practitioner should be cautious and not to increase the Vitamin D dose based on the iPTH level. Instead, they should use the $25(\mathrm{OH}) \mathrm{D}$ to guide replacement therapy. The Endocrine Society recommends a minimum level of $25(\mathrm{OH}) \mathrm{D}$ of 30 $\mathrm{ng} / \mathrm{mL}$. A level between 20 and 30 is considered insufficient, while those below 20 as deficient. ${ }^{12}$

\section{Will the patient get vitamin D toxicity?}

If guidelines are adhered to strictly, toxicity from vitamin $D$ is rare. Caution should be the rule when treating certain patients with chronic granulomatous diseases and certain lymphomas, which produce 1-alphahydroxylase in their macrophages and consequently increase the production of $1,25(\mathrm{OH}) 2 \mathrm{D}$ in an unregulated manner.13

\section{CONCLUSION}

Vitamin D deficiency is not a diagnosis of exclusion. A good history, physical examination, and relevant laboratory tests will lead to a timely diagnosis and appropriate therapy. Prevention saves healthcare costs and reduces morbidity and mortality. High-risk groups will need greater attention and will benefit most from intervention. Proximal muscle pelvic girdle myopathy in the correct context should prompt testing for $25(\mathrm{OH}) \mathrm{D}$ levels utilising reliable assays, which is the ideal substance in the blood that reflects body vitamin $D$ stores. Testing for $1,25(\mathrm{OH}) 2 \mathrm{D}$ is not useful as its half life is short; does not reflect body stores of vitamin D; and is often normal, even in severe deficiency states. Unnecessary investigations like nuclear bone scans can be very misleading and should be discouraged. Treatment is readily available, safe, and effective, even when the disease is advanced. A full recovery is a rule, as seen in the above examples. Finally, it is vital to educate not only the general public but also healthcare professionals, both generalists and specialists, to enable them to recognise vitamin $D$ deficiency in a timely manner and manage patients appropriately to improve healthcare outcomes.

\section{References}

1. Harinarayan $\vee$ et al. Vitamin D status and sun exposure in India. Dermatoendocrinol. 2013;5(1):130-41.

2. Fox A et al. Vitamin D status after gastric bypass or sleeve gastrectomy over 4 years of follow-up. Obes Surg. 2020;30(4):1473-81.

3. Holick MF. Vitamin D: a d-lightful solution for health. J Investig Med. 2011;59(6):872-80

4. Maurya VK, Aggarwal M. Factors influencing the absorption of vitamin D in GIT: an overview. J Food Sci Technol. 2017;54(12):3753-65.

5. Latham CM et al. Vitamin D promotes skeletal muscle regeneration and mitochondrial health. Front Physiol. 2021;12:660498.
6. Sanders KM et al. Vitamin D deficiency and its role in musclebone interactions in the elderly. Curr Osteoporos Rep. 2014;12(1):74-81.

7. Nunes $\mathrm{G}$ et al. Adult coeliac disease: the importance of extraintestinal manifestations. GE Port J Gastroenterol. 2017;24(6):292.

8. Chakhtoura MT et al. Guidelines on vitamin $D$ replacement in bariatric surgery: Identification and systematic appraisal. Metabolism. 2016;65(4):586-97.

9. Gorman S et al. High-dose intramuscular vitamin D provides long-lasting moderate increases in serum 25-hydroxvitamin D levels and shorter-term changes in plasma calcium. J AOAC Int.

\section{7;100(5):1337-44}

10. De Niet $\mathrm{S}$ et al. A randomised study to compare a monthly to a daily administration of vitamin D3 supplementation. Nutrients. 2018;10(6):659

11. Kennel KA et al. Vitamin D deficiency in adults: when to test and how to treat. Mayo Clin Proc. 2010;85(8):752-8.

12. Holick MF et al. Evaluation, treatment, and prevention of vitamin D deficiency: an Endocrine Society clinical practice guideline. J Clin Endocrinol Metab. 2011;96(7):1911-30.

13. Sarathi $\vee$ et al. High-dose vitamin D supplementation precipitating hypercalcemic crisis in granulomatous disorders. Indian J Endocrinol Metab. 2017;21(6):815-9. 